\title{
非自治线性差分方程全局吸引性中的 若干问题
}

\author{
庭建设郭志明
}

(广州大学理学院, 广州 510405)

摘要 旨在解决非自治差分方程

$$
x_{n+1}-x_{n}+P_{n} x_{n-k_{n}}=0, \quad n \in \mathbb{Z}(0)
$$

零解全局吸引性的若千问题，其中 $\left\{P_{n}\right\}$ 是非负实数序列， $\left\{k_{n}\right\}$ 是非负整数序 列, 并且当 $n \rightarrow \infty$ 时, $n-k_{n} \rightarrow \infty$.

\section{关键词 差分方程 全局吸引性 非自治}

\section{1 引言}

记 $\mathbb{Z}$ 及 $\mathbb{R}$ 分别为整数集与实数集. 对于任意的 $a, b \in \mathbb{Z}$, 定义 $\mathbb{Z}(a) \triangleq\{a, a+$ $1, \cdots\}$, 并且当 $a \leqslant b$ 时, $\mathbb{Z}(a, b) \triangleq\{a, a+1, \cdots, b\}$. 考虑非自治线性差分方程

$$
x_{n+1}-x_{n}+P_{n} x_{n-k_{n}}=0, \quad n \in \mathbb{Z}(0),
$$

其中 $\left\{P_{n}\right\}$ 为非负实数序列, $\left\{k_{n}\right\}$ 是非负整数序列, 且满足 $n \rightarrow \infty$ 时, $n-k_{n} \rightarrow$ $\infty$.

关于差分方程的定性性质, 最近已有大量的文献进行了广泛的讨论 ${ }^{[1 \sim 4]}$. 对 线性差分方程, 如方程 (1.1), 许多作者研究了其解的振动性、渐近性与稳定性. 例如, 关于振动性的结果可参见文献 [3,5 10]; 关于渐近性与稳定性的结果可参 见文献 $[1,3,8,11 \sim 14]$; 对非线性情形，可参阅文献 [1, 3 5, 15 17] 等.

在 $k_{n} \equiv k$ 的情形, 文献 [8] (也可参见文献 [3]) 证明了: 如果

$$
P_{n}>0, \quad \limsup _{n \rightarrow \infty} \sum_{i=n-k}^{n} P_{i}<1
$$

2003-02-24 收稿

* 教育部跨世纪优秀人才培养计划与高校博士学科点专项科研基金资助项目 
以及

$$
\sum_{n=0}^{\infty} P_{n}=\infty
$$

成立, 则方程 (1.1) 的任一解当 $n \rightarrow \infty$ 时均趋于零.

这是现有文献中, 关于方程 (1.1) 解的渐近性的第 1 个结果. 注意到 (1.2) 式 中和的项数为 $k+1$, 我们希望将其和的项数减为 $k$, 因此有如下的问题:

问题 1 假定 (1.3) 式成立, (1.2) 式能否改进为

$$
\limsup _{n \rightarrow \infty} \sum_{i=n-k}^{n-1} P_{i}<1 ?
$$

文献 [11] 证明了 (1.2) 式可以改进为

$$
\limsup _{n \rightarrow \infty} \sum_{i=n-k}^{n} P_{i}<\frac{3}{2}+\frac{1}{2(k+1)} .
$$

显而易见, (1.4) 与 (1.5) 式是相互独立的, 并且条件 (1.5) 是对 (1.2) 式的一 个很大改进. 但 (1.5) 式能否继续改进仍是一个尚未解决的问题, 因此, 我们有 如下的问题:

问题 2 (1.5) 式中的上界 $\frac{3}{2}+\frac{1}{2(k+1)}$ 可否用大于它的某个常数来代替?

对于 $k_{n}$ 是变时滞的情形, 文献 [14] 证明了: 当 $\left\{k_{n}\right\}$ 无界时, 如果 (1.3) 式 成立, 并且

$$
\limsup _{n \rightarrow \infty} \sum_{i=n-k_{n}}^{n} P_{i}<\frac{3}{2}
$$

则方程 (1.1) 的每个解当 $n \rightarrow \infty$ 时趋于零.

类似于问题 2 , 我们有

问题 3 当 $\left\{k_{n}\right\}$ 无界时, 条件 (1.6) 中的上界 $\frac{3}{2}$ 可否进一步改进?

本文的目的是详细讨论并回答上述 3 个问题.

方程 (1.1) 可以作为时滞微分方程

$$
x^{\prime}(t)+P(t) x(t-\tau(t))=0, \quad t \geqslant 0
$$

的离散化形式, 其中 $P, \tau \in C([0, \infty),[0, \infty))$. 关于方程 (1.7), 其解的渐近行为已 有许多文献进行过讨论, 例如文献 [18 21]. 对方程 (1.1) 的许多研究成果大多都 与方程 (1.7) 的相应结果的离散情形类似, 可参见文献 $[3,6,7,16]$.

为方便起见，本文采用如下约定:

$$
\sum_{n=i}^{j} P_{n} \equiv 0 \text {, 当 } j \leqslant i-1 \text { 时. }
$$

\section{2 问题 1}

本节将回答问题 1. 下面的定理说明: 不论当 $\left\{k_{n}\right\}$ 是有界或无界, 还是常值 或可变时，问题 1 的回答都是肯定的. 
定理 2.1 假设 (1.3) 式成立, 对于任意的 $i \in \mathbb{Z}(0),\left\{k_{n}\right\}_{n=i}^{\infty} \not \equiv 0$, 并且

$$
\limsup _{n \rightarrow \infty} \sum_{i=n-k_{n}}^{n-1} P_{i}<1,
$$

则 (1.1) 式的每个解当 $n \rightarrow \infty$ 时都趋于零.

证 假设 $\left\{x_{n}\right\}$ 是方程 (1.1) 的解, 将证明

$$
\lim _{n \rightarrow \infty} x_{n}=0 .
$$

如果 $\left\{x_{n}\right\}$ 是最终非负或最终非正的, 则由 (1.3) 式及 $\left\{x_{n}\right\}$ 的单调性易知 (2.2) 式 成立. 下面考虑 $\left\{x_{n}\right\}$ 是振动的情形, 即 $\left\{x_{n}\right\}$ 既非最终正解, 亦非最终负解. 由 (2.1) 式，存在正整数 $N$ 及正常数 $\alpha<1$, 使得

$$
\sum_{i=n-k_{n}}^{n-1} P_{i} \leqslant \alpha, \quad n \in n \in \mathbb{Z}(N) .
$$

首先证明 $\left\{x_{n}\right\}$ 的有界性. 假设 $\left\{x_{n}\right\}$ 是无界的, 则可以选取 $n^{*} \in \mathbb{Z}(N+1)$, 使得 $n^{*}>k_{n^{*}}$ 时,

$$
\left|x_{n^{*}}\right|>\max _{n \in \mathbb{Z}\left(0, n^{*}-1\right)}\left\{\left|x_{n}\right|\right\}
$$

不失一般性, 设 $x_{n^{*}}>0$, 则 $x_{n^{*}}-x_{n^{*}-1}>0$. 由 (1.1) 式, $x_{n^{*}-1-k_{n^{*}-1}}<0$. 将 (1.1) 式两边从 $n^{*}-1-k_{n^{*}-1}$ 到 $n^{*}-1$ 相加, 有

$$
\begin{aligned}
x_{n^{*}} & =x_{n^{*}-1-k_{n^{*}-1}}-\sum_{i=n^{*}-1-k_{n^{*}-1}}^{n^{*}-1} P_{i} x_{i-k_{i}} \\
& =\left(1-P_{n^{*}-1}\right) x_{n^{*}-1-k_{n^{*}-1}}-\sum_{i=n^{*}-1-k_{n^{*}-1}}^{n^{*}-2} P_{i} x_{i-k_{i}} \\
& \leqslant\left(1-P_{n^{*}-1}\right) x_{n^{*}-1-k_{n^{*}-1}}+x_{n^{*}} \sum_{i=n^{*}-1-k_{n^{*}-1}}^{n^{*}-2} P_{i} .
\end{aligned}
$$

由 (2.3) 式易得, $P_{n^{*}-1} \leqslant \alpha<1$, 因此有

$$
x_{n^{*}}<\alpha x_{n^{*}}
$$

这是不可能的, 故 $\left\{x_{n}\right\}$ 有界.

现令

$$
\mu=\limsup _{n \rightarrow \infty}\left|x_{n}\right|
$$

则 $0 \leqslant \mu<\infty$. 只需证明 $\mu=0$. 对于任给的 $\varepsilon>0$, 存在 $m^{*} \in \mathbb{Z}(N+1)$, 使得

$$
\left|x_{n-k_{n}}\right|<\mu+\varepsilon, \quad n \in \mathbb{Z}\left(m^{*}\right) .
$$


因为 $\left\{x_{n}\right\}$ 是振动的, 故存在整数序列 $\left\{n_{i}\right\}$, 使得 $n_{i} \in \mathbb{Z}\left(m^{*}\right), x_{n_{i}} \cdot \triangle x_{n_{i}-1}>0$, 并且当 $i \rightarrow \infty$ 时, $n_{i} \rightarrow \infty,\left|x_{n_{i}}\right| \rightarrow \mu$. 我们将证明

$$
\left|x_{n_{i}}\right|<\alpha(\mu+\varepsilon), i \in \mathbb{Z}(1) \text {. }
$$

不失一般性, 假设 $x_{n_{i}}>0$, 则 $x_{n_{i}}-x_{n_{i}-1}>0$. 由 (1.1) 式, $x_{n_{i}-1-k_{n_{i}-1}}<0$. 考虑到 (2.3) 及 $(2.4)$ 式, 有

$$
\begin{aligned}
\left|x_{n_{i}}\right| & =x_{n_{i}}=x_{n_{i}-1-k_{n_{i}-1}}+\sum_{j=n_{i}-1-k_{n_{i}-1}}^{n_{i}-1}\left(x_{j+1}-x_{j}\right) \\
& =x_{n_{i}-1-k_{n_{i}-1}}-\sum_{j=n_{i}-1-k_{n_{i}-1}}^{n_{i}-1} P_{j} x_{j-k_{j}} \\
& =\left(1-P_{n_{i}-1}\right) x_{n_{i}-1-k_{n_{i}-1}}-\sum_{j=n_{i}-1-k_{n_{i}-1}}^{n_{i}-2} P_{j} x_{j-k_{j}} \\
& <(\mu+\varepsilon) \sum_{j=n_{i}-1-k_{n_{i}-1}}^{n_{i}-2} P_{j} \leqslant \alpha(\mu+\varepsilon) .
\end{aligned}
$$

这证明了 (2.5) 式成立. 令 $i \rightarrow \infty$, 并注意到 $\varepsilon$ 的任意性, 可得

$$
\mu \leqslant \alpha \mu .
$$

由于 $\alpha<1$, 得 $\mu=0$. 证毕.

推论 2.2 假设 (1.3) 式成立, 对于任意的 $i \in \mathbb{Z}(0),\left\{k_{n}\right\}_{n=i}^{\infty} \not \equiv 0$, 并且存在正 整数 $k$, 使得 $k_{n} \leqslant k, n \in \mathbb{Z}(0)$, 以及

$$
\limsup _{n \rightarrow \infty} P_{n}<\frac{1}{k}
$$

则方程 (1.1) 的每个解当 $n \rightarrow \infty$ 时都趋于零.

当 $k_{n} \equiv 1$ 并且 $P_{n} \equiv P$ 时, (2.6) 式成为

$$
P<1,
$$

这也是方程 (1.1) 零解全局吸引性的必要条件 ${ }^{[12]}$.

\section{3 问题 2}

本节通过构造反例的方法, 证明 (1.5) 式中的上界 $\frac{3}{2}+\frac{1}{2(k+1)}$ 是最好的选择. 当 $k=0$ 时, (1.5) 式成为

$$
\limsup _{n \rightarrow \infty} P_{n}<2 .
$$

此上界 2 是不可改进的, 因为当 $P_{n} \equiv P, k=0$ 时, (3.1) 式是方程 (1.1) 的每个解 当 $n \rightarrow \infty$ 时趋于零的充分必要条件 (参见文献 [12]).

现取定 $k \in \mathbb{Z}(1), c>0$ 为给定的常数. 考虑非自治差分方程

$$
y_{n+1}-y_{n}+P_{n} y_{n-k}=0, \quad n \in \mathbb{Z}(0),
$$


其中 $\left\{P_{n}\right\}$ 定义为

$$
P_{n}=\left\{\begin{array}{cll}
\frac{1}{k+1}, & \text { 当 } & n \in \mathbb{Z}(i(3 k+1), k-1+i(3 k+1)) \\
& & \cup \mathbb{Z}(k+1+i(3 k+1), 2 k+i(3 k+1)) ; \\
c, & \text { 当 } & n=k+i(3 k+1) ; \\
0, & \text { 当 } & n \in \mathbb{Z}(2 k+1+i(3 k+1), 3 k+i(3 k+1)),
\end{array}\right.
$$

$i \in \mathbb{Z}(0)$. 注意到

$$
\sup _{n \in \mathbb{Z}(k)} \sum_{j=n-k}^{n} P_{j}=c+\frac{k}{k+1}
$$

这样由文献 [11] 可知, 如果

$$
c+\frac{k}{k+1}<\frac{3}{2}+\frac{1}{2(k+1)},
$$

或等价地

$$
c<\frac{3}{2}-\frac{2 k-1}{2(k+1)}=\frac{k+4}{2(k+1)},
$$

则方程 (3.2) 的每个解当 $n \rightarrow \infty$ 时趋于零.

下面的定理表明: 如果 (3.4) 式不成立, 则存在方程 (3.2) 的一个解, 当 $n \rightarrow \infty$ 时不趋于零. 这说明 (1.5) 式的上界 $\frac{3}{2}+\frac{1}{2(k+1)}$ 不能再进一步改进.

定理 3.1 假设

$$
c \geqslant \frac{k+4}{2(k+1)}
$$

则方程 (3.2) 存在一个解, 当 $n \rightarrow \infty$ 时不趋于零.

证 设 $y_{n}$ 是方程 (3.2) 满足如下初始条件的解:

$$
y_{i}=\varepsilon, \quad i \in \mathbb{Z}(-k, 0),
$$

其中 $\varepsilon>0$ 是给定的常数. 由方程 (3.2), 有

$$
y_{n+1}-y_{n}=-\varepsilon P_{n}, \quad n \in \mathbb{Z}(0, k) .
$$

由此可得

$$
y_{n}=\varepsilon-\varepsilon \sum_{j=0}^{n-1} P_{j}=\varepsilon\left(1-\frac{n}{k+1}\right), n \in \mathbb{Z}(0, k)
$$

及

$$
y_{k+1}=y_{k}-\varepsilon c=\varepsilon\left(1-\frac{k}{k+1}-c\right)=\varepsilon\left(\frac{1}{k+1}-c\right) .
$$

又由 (3.2) 与 (3.7) 式, 有

$$
y_{n+1}-y_{n}=-\varepsilon\left(1-\frac{n-k}{k+1}\right) \frac{1}{k+1}, \quad n \in \mathbb{Z}(k+1,2 k) .
$$


在 (3.9) 式两边从 $k+1$ 到 $2 k$ 相加, 并借助于 (3.8) 式, 得到

$$
\begin{aligned}
y_{2 k+1} & =y_{k+1}-\varepsilon \frac{1}{k+1} \sum_{n=k+1}^{2 k}\left(1-\frac{n-k}{k+1}\right) \\
& =-\varepsilon\left[c+\frac{k-2}{2(k+1)}\right] \stackrel{\text { def }}{=} \varepsilon_{1} .
\end{aligned}
$$

进一步, 由 $\left\{P_{n}\right\}$ 的定义, 得到

$$
y_{n} \equiv \varepsilon_{1}, \quad n \in \mathbb{Z}(2 k+1,3 k+1) .
$$

定义序列 $\left\{\varepsilon_{i}\right\}$ 如下:

$$
\varepsilon_{0}=\varepsilon, \varepsilon_{i+1}=-\varepsilon_{i}\left[c+\frac{k-2}{2(k+1)}\right], \quad i \in \mathbb{Z}(0) .
$$

重复上述论证，应用归纳法，可得

$$
y_{n}=\varepsilon_{i+1}, \quad n \in \mathbb{Z}(2 k+1+i(3 k+1),(i+1)(3 k+1)), \quad i \in \mathbb{Z}(0) .
$$

考虑到 (3.5) 式, 有

$$
\left|\varepsilon_{i+1}\right|=\left|\varepsilon_{i}\right|\left|c+\frac{k-2}{2(k+1)}\right| \geqslant\left|\varepsilon_{i}\right|, \quad i \in \mathbb{Z}(0) .
$$

这说明序列 $\left\{\left|\varepsilon_{i}\right|\right\}$ 是单调不减的正数序列, 因此 $\left|\varepsilon_{i}\right|$ 当 $n \rightarrow \infty$ 时不趋于零. 由 (3.12) 式, 知 $\left\{y_{n}\right\}$ 当 $n \rightarrow \infty$ 时不趋于零. 证毕.

\section{4 问题 3}

本节研究问题 3. 通过构造另一个反例, 证明条件 (1.6) 中的上界 $\frac{3}{2}$ 也是最好 的选择.

定义

$$
\xi_{i}=5 i+\frac{3(i-1) i}{2}, \quad \eta_{i}=\frac{3(i+1)(i+2)}{2}-2, \quad i \in \mathbb{Z}(0),
$$

则 $\left\{\xi_{i}\right\}$ 与 $\left\{\eta_{i}\right\}$ 单调递增, 当 $i \rightarrow \infty$ 时均趋于无穷大, 并且满足 $\eta_{i} \in \mathbb{Z}\left(\xi_{i}, \xi_{i+1}-1\right)$. 令 $P>0$. 当 $i \in \mathbb{Z}(0)$ 并且 $n \in \mathbb{Z}\left(\xi_{i}, \xi_{i+1}-1\right)$ 时, 令 $k_{n}=i+1$. 再设

$$
P_{n}=\left\{\begin{array}{cl}
\frac{1}{i+2}, & \text { 当 } n \in \mathbb{Z}\left(\xi_{i}, \eta_{i}-1\right) \cup \mathbb{Z}\left(\eta_{i}+1,2 \eta_{i}-\xi_{i}\right) ; \\
P+\frac{3}{2(i+2)}, & \text { 当 } n=\eta_{i} ; \\
0, & \text { 当 } n \in \mathbb{Z}\left(2 \eta_{i}-\xi_{i}+1, \xi_{i+1}-1\right),
\end{array}\right.
$$

其中 $i \in \mathbb{Z}(0)$.

不难验证 $\left\{k_{n}\right\}$ 是无界的非负整数序列, 且满足当 $n \rightarrow \infty$ 时, $n-k_{n} \rightarrow \infty$.

考虑具有无界时滞的差分方程

$$
z_{n+1}-z_{n}+P_{n} z_{n-k_{n}}=0, \quad n \in \mathbb{Z}(0),
$$

经过简单计算，得

$$
\limsup _{n \rightarrow \infty} \sum_{j=n-k_{n}}^{n} P_{j}=P+1
$$


由文献 [14] 可知, 如果 $P<\frac{1}{2}$, 则方程 (4.1) 的每个解当 $n \rightarrow \infty$ 时趋于零. 下面将证明: 如果 $P \geqslant \frac{1}{2}$, 则存在方程 (4.1) 的一个解, 当 $n \rightarrow \infty$ 时不趋于零.

定理 4.1 假设 $P \geqslant \frac{1}{2}$, 则必存在方程 (4.1) 的一个解, 当 $n \rightarrow \infty$ 时不趋于 零.

证 设 $\left\{z_{n}\right\}$ 是方程 (4.1) 满足初始条件: $z_{n} \equiv \varepsilon(n \in \mathbb{Z}(-1,0))$ 的解, 其中 $\varepsilon>0$ 为某个常数.

由方程 (4.1), 有

$$
z_{n+1}-z_{n}=-\varepsilon P_{n}, \quad n \in \mathbb{Z}\left(\xi_{0}, \eta_{0}\right),
$$

则对于任意的 $n \in \mathbb{Z}\left(\xi_{0}, \eta_{0}+1\right)$,

$$
z_{n}=z_{0}-\varepsilon \sum_{j=0}^{n-1} P_{j}=\varepsilon\left(1-\sum_{j=0}^{n-1} P_{j}\right) .
$$

因此 $z_{1}=\varepsilon\left(1-P_{0}\right)=\varepsilon\left(1-\frac{1}{2}\right)=\frac{1}{2} \varepsilon, z_{2}=z_{\eta_{0}+1}=\varepsilon\left(1-P_{0}-P_{1}\right)=\varepsilon\left(1-\frac{1}{2}-P-\frac{3}{4}\right)=$ $-\varepsilon\left(P+\frac{1}{4}\right)$.

仍由方程 (4.1), 可知

$$
z_{3}=z_{2 \eta_{0}-\xi_{0}+1}=z_{2}-P_{2} z_{1}=-\varepsilon\left(P+\frac{1}{4}\right)-\frac{1}{2} \cdot \frac{1}{2} \varepsilon=-\varepsilon\left(P+\frac{1}{2}\right) .
$$

因为对于任意的 $n \in \mathbb{Z}\left(2 \eta_{0}-\xi_{0}+1, \xi_{1}-1\right), P_{n}=0$, 有

$$
z_{n} \equiv z_{3}=-\varepsilon\left(P+\frac{1}{2}\right), \quad n \in \mathbb{Z}\left(2 \eta_{0}-\xi_{0}+1, \xi_{1}\right) .
$$

定义序列 $\left\{\varepsilon_{i}\right\}$ 如下:

$$
\varepsilon_{0}=\varepsilon, \quad \varepsilon_{i}=-\left(P+\frac{1}{2}\right) \varepsilon_{i-1}, \quad i \in \mathbb{Z}(1) .
$$

由于 $P \geqslant \frac{1}{2}$, 易知当 $i \rightarrow \infty$ 时, $\left\{\varepsilon_{i}\right\}$ 不以零为极限. 根据 (4.4) 式, 有

$$
z_{n} \equiv \varepsilon_{1}, \quad n \in \mathbb{Z}\left(2 \eta_{0}-\xi_{0}+1, \xi_{1}\right) .
$$

一般地, 证明

$$
z_{n} \equiv \varepsilon_{i+1}, \quad n \in \mathbb{Z}\left(2 \eta_{i}-\xi_{i}+1, \xi_{i+1}\right) .
$$

由 (4.6) 式可知，当 $i=0$ 时，(4.7) 式成立. 下证: 当 $i=1$ 时，(4.7) 式成立. 事 实上, 由 (4.1) 及 (4.6) 式, 有

$$
z_{n+1}-z_{n}=-\varepsilon_{1} P_{n}, \quad n \in \mathbb{Z}\left(\xi_{1}, \eta_{1}\right) .
$$

由此可得

$$
z_{n}=z_{\xi_{1}}-\varepsilon_{1} \sum_{j=\xi_{1}}^{n-1} P_{j}, \quad n \in \mathbb{Z}\left(\xi_{1}, \eta_{1}+1\right) .
$$

注意到 $\left\{P_{n}\right\}$ 的定义，有

$$
z_{n}=\varepsilon_{1}\left[1-\left(n-\xi_{1}\right) \cdot \frac{1}{3}\right], \quad n \in \mathbb{Z}\left(\xi_{1}, \eta_{1}\right)
$$


及

$$
\begin{aligned}
z_{\eta_{1}+1} & =\varepsilon_{1}\left[1-\left(\eta_{1}-\xi_{1}\right) \cdot \frac{1}{3}-P-\frac{1}{2}\right] \\
& =\varepsilon_{1}\left(1-\frac{2}{3}-P-\frac{1}{2}\right)=\varepsilon_{1}\left(-\frac{1}{6}-P\right) .
\end{aligned}
$$

由于对任意的 $n \in \mathbb{Z}\left(\eta_{1}+1,2 \eta_{1}-\xi_{1}\right), n-k_{n} \in \mathbb{Z}\left(\xi_{1}, \eta_{1}\right)$, 从而

$$
\begin{aligned}
z_{n+1}-z_{n} & =-\varepsilon_{1} P_{n}\left[1-\left(n-k_{n}-\xi_{1}\right) \cdot \frac{1}{3}\right]=-\varepsilon_{1} \cdot \frac{1}{3} \cdot\left[1-(n-2-5) \cdot \frac{1}{3}\right] \\
& =-\frac{\varepsilon_{1}}{9}(10-n), \quad n \in \mathbb{Z}\left(\eta_{1}+1,2 \eta_{1}-\xi_{1}\right)=\mathbb{Z}(8,9),
\end{aligned}
$$

并且有

$$
z_{n}=z_{\eta_{1}+1}-\frac{\varepsilon_{1}}{9} \sum_{j=n_{1}+1}^{n-1}(10-j), \quad n \in \mathbb{Z}(8,10) .
$$

这样得到

$$
\begin{aligned}
z_{2 \eta_{1}-\xi_{1}+1} & =z_{10}=\varepsilon_{1}\left(-\frac{1}{6}-P\right)-\frac{\varepsilon_{1}}{9} \sum_{j=8}^{9}(10-j) \\
& =-\varepsilon_{1}\left(P+\frac{1}{2}\right)=\varepsilon_{2}
\end{aligned}
$$

并进一步有

$$
z_{n} \equiv \varepsilon_{2}, \quad n \in \mathbb{Z}\left(2 \eta_{1}-\xi_{1}+1, \xi_{2}\right) .
$$

此即说明当 $i=1$ 时, (4.7) 式成立.

现假设对某个 $i_{0} \in \mathbb{Z}(0)$, (4.7) 式成立，即

$$
z_{n} \equiv \varepsilon_{i_{0}+1}, \quad n \in \mathbb{Z}\left(2 \eta_{i_{0}}-\xi_{i_{0}}+1, \xi_{i_{0}+1}\right) .
$$

由归纳法, 只需证明

$$
z_{n} \equiv \varepsilon_{i_{0}+2}, \quad n \in \mathbb{Z}\left(2 \eta_{i_{0}+1}-\xi_{i_{0}+1}+1, \xi_{i_{0}+2}\right) .
$$

由 (4.1) 和 (4.12) 式, 有

$$
z_{n+1}-z_{n}=-\varepsilon_{i_{0}+1} P_{n}, \quad n \in \mathbb{Z}\left(\xi_{i_{0}+1}, \eta_{i_{0}+1}\right) .
$$

对于 $n \in \mathbb{Z}\left(\xi_{i_{0}+1}, \eta_{i_{0}+1}+1\right)$, 将 (4.14) 式两边从 $\xi_{i_{0}+1}$ 到 $n-1$ 相加, 可得

$$
z_{n}=z_{\xi_{i_{0}+1}}-\varepsilon_{i_{0}+1} \sum_{j=\xi_{i_{0}+1}}^{n-1} P_{j}=\varepsilon_{i_{0}+1}\left(1-\sum_{j=\xi_{i_{0}+1}}^{n-1} P_{j}\right),
$$

因而

$$
\begin{gathered}
z_{n}=\varepsilon_{i_{0}+1}\left[1-\left(n-\xi_{i_{0}+1}\right) \cdot \frac{1}{i_{0}+3}\right], \quad n \in \mathbb{Z}\left(\xi_{i_{0}+1}, \eta_{i_{0}+1}\right), \\
\text { www.scichina.com }
\end{gathered}
$$


并且

$$
\begin{aligned}
z_{\eta_{i_{0}+1}+1} & =\varepsilon_{i_{0}+1}\left[1-\left(\eta_{i_{0}+1}-\xi_{i_{0}+1}\right) \cdot \frac{1}{i_{0}+3}-P_{\eta_{i_{0}+1}}\right] \\
& =\varepsilon_{i_{0}+1}\left[1-\frac{i_{0}+2}{i_{0}+3}-P-\frac{3}{2\left(i_{0}+3\right)}\right] \\
& =-\varepsilon_{i_{0}+1}\left[P+\frac{1}{2\left(i_{0}+3\right)}\right] .
\end{aligned}
$$

由于对任意的 $n \in \mathbb{Z}\left(\eta_{i_{0}+1}+1,2 \eta_{i_{0}+1}-\xi_{i_{0}+1}\right)$, 有 $n-k_{n} \in \mathbb{Z}\left(\xi_{i_{0}+1}, \eta_{i_{0}+1}\right)$, 再由 (4.1) 与 (4.15) 式可得

$$
\begin{aligned}
z_{n+1}-z_{n} & =-P_{n} \varepsilon_{i_{0}+1}\left(1-\frac{n-k_{n}-\xi_{i_{0}+1}}{i_{0}+3}\right) \\
& =-\frac{\varepsilon_{i_{0}+1}}{i_{0}+3}\left(1-\frac{n-i_{0}-2-\xi_{i_{0}+1}}{i_{0}+3}\right) \\
& =-\frac{\varepsilon_{i_{0}+1}}{\left(i_{0}+3\right)^{2}}\left(2 i_{0}+5+\xi_{i_{0}+1}-n\right) .
\end{aligned}
$$

由上式及 (4.16) 式, 对于任意的 $n \in \mathbb{Z}\left(\eta_{i_{0}+1}+1,2 \eta_{i_{0}+1}-\xi_{i_{0}+1}+1\right)$, 有

$$
\begin{aligned}
z_{n} & =z_{\eta_{i_{0}+1}+1}-\frac{\varepsilon_{i_{0}+1}}{\left(i_{0}+3\right)^{2}} \sum_{j=\eta_{i_{0}+1}+1}^{n-1}\left(2 i_{0}+5+\xi_{i_{0}+1}-j\right) \\
& =-\varepsilon_{i_{0}+1}\left[P+\frac{1}{2\left(i_{0}+3\right)}\right]-\frac{\varepsilon_{i_{0}+1}}{\left(i_{0}+3\right)^{2}} \sum_{j=\eta_{i_{0}+1}+1}^{n-1}\left(2 i_{0}+5+\xi_{i_{0}+1}-j\right),
\end{aligned}
$$

因此

$$
\begin{aligned}
& z_{2 \eta_{i_{0}+1}-\xi_{i_{0}+1}}+1 \\
=-\varepsilon_{i_{0}+1} & {\left[P+\frac{1}{2\left(i_{0}+3\right)}\right]-\frac{\varepsilon_{i_{0}+1}}{\left(i_{0}+3\right)^{2}} \sum_{j=\eta_{i_{0}+1}+1}^{2 \eta_{i_{0}+1}-\xi_{i_{0}+1}}\left(2 i_{0}+5+\xi_{i_{0}+1}-j\right) } \\
=-\varepsilon_{i_{0}+1} & {\left[P+\frac{1}{2\left(i_{0}+3\right)}+\frac{1}{\left(i_{0}+3\right)^{2}}\left(2 i_{0}+5+\xi_{i_{0}+1}\right)\left(\eta_{i_{0}+1}-\xi_{i_{0}+1}\right)\right.} \\
& \left.\quad-\frac{1}{2\left(i_{0}+3\right)^{2}}\left(3 \eta_{i_{0}+1}-\xi_{i_{0}+1}+1\right)\left(\eta_{i_{0}+1}-\xi_{i_{0}+1}\right)\right] \\
= & -\varepsilon_{i_{0}+1}\left(P+\frac{1}{2}\right) \\
= & \varepsilon_{i_{0}+2} .
\end{aligned}
$$

因为对任意的 $n \in \mathbb{Z}\left(2 \eta_{i_{0}+1}-\xi_{i_{0}+1}+1, \xi_{i_{0}+2}-1\right)$, 有 $P_{n} \equiv 0$, 故由 (4.1) 式可知

$$
z_{n} \equiv \varepsilon_{i_{0}+2}, \quad n \in \mathbb{Z}\left(2 \eta_{i_{0}+1}-\xi_{i_{0}+1}+1, \xi_{i_{0}+2}\right) .
$$

这证明了 (4.13) 式成立, 因此 (4.7) 式成立. 在 (4.7) 式中令 $i \rightarrow \infty$, 并注意到 $\left\{\varepsilon_{i}\right\}$ 不以零为极限, 由方程 (4.1) 的解知 $\left\{z_{n}\right\}$ 当 $n \rightarrow \infty$ 时不趋于零. 证毕. 


\section{参考文献}

1 Agarwal R P. Difference Equations and Inequalities: Theory, Method and Applications. New York: Marcel Dekker, 2000

2 Elaydi S N. An Introduction to Difference Equations. 2nd ed. New York: Springer-Verlag, 1999

3 Györi I, Ladas G. Oscillation Theory of Delay Differential Equations with Applications. Oxford: Oxford University Press, 1991

4 Kocic V LJ, Ladas G. Global Behavior of Nonlinear Difference Equations of Higher Order with Applications. Boston: Kluwer Academic Publishers, 1993

5 Chen M P, Yu J S. Oscillation and global attractivity in a delay logistic difference equation. J Difference Equations Appl, 1995, 1: 227 237

6 Erbe L H, Zhang B G. Oscillation of discrete analogue of delay equations. Differential and Integral Equations, 1989, 2: 300 309

7 Ladas G, Philos CH G, Sficas Y G. Sharp conditions for the oscillation of delay difference equations. J Applic Math Simulation, 1989, 2: 101 109

8 Ladas G, Qian C, Vlahos P N, et al. Stability of solutions of linear nonautonomous difference equations. Appl Anal, 1991, 41: 183 191

9 Philos CH G. Oscillation of some difference equations. Funkcialaj Ekvacioj, 1991, 34: 157 172

10 Philos CH G. Oscillation in a nonautonomous delay logistic difference equation. Proc Edinburgh Math Soc, 1992, 35: 121 131

11 Erbe L H, Xia H, Yu J S. Global stability of a linear nonautonomous delay difference equations. J Difference Equations Appl, 1995, 1: 151 161

12 Kuruklis S A. The asymptotic stability of $x_{n+1}-a x_{n}+b x_{n-k}=0$. J Math Anal Appl, 1994, 188: 719 731

13 Yu J S. Asymptotic stability for a linear difference equation with variable delay. Computers Math Applic, 1998, 36(10-12): 203 210

14 Zhang B G, Tian C J, Wong P J Y. Global attractivity of difference equations with variable delay. Dynamics of Continuous, Discrete and Impulsive Systems, 1999, 6: 307 317

15 Kocic V LJ, Ladas G. Global attractivity in nonlinear delay difference equations. Proc Amer Math Soc, 1992, 115: 1083 1088

16 Matsunaga H, Hara T, Sakata S. Global attractivity for a nonlinear difference equation with variable delay. Computers Math Applic, 2001, 41: 543 551

17 Zhou Z, Zhang Q. Uniform stability of nonlinear difference systems. J Math Anal Appl, 1998, 225: 486 500

18 Joseph So W H, Yu J S, Chen M P. Asymptotic stability for scalar delay differential equations. Funkcialaj Ekvacioj, 1996, 39: 1 17

19 Kuang Y. Delay Differential Equations with Applications in Population Dynamics. San Diego: Academic Press, 1993

20 Yoneyama T. The $\frac{3}{2}$ stability theorem for one-dimensional delay-differential equations with unbounded delay. J Math Anal Appl, 1992, 165: 133 143

21 Yorke J A. Asymptotic stability for one-dimensional delay-differential equations. J Differential Equations, 1970, 7: 189 202 